\title{
Remoção de braquetes cerâmicos com alicate de How associado à broca diamantada - avaliação da topografia do esmalte
}

\author{
Matheus Melo Pithon*, Márlio Vinícius de Oliveira**, Antônio Carlos de Oliveira Ruellas***
}

\begin{abstract}
Resumo
Objetivo: avaliar a topografia do esmalte dentário após a descolagem de braquetes cerâmicos Allure (GAC/Dentsply) através de dois diferentes métodos. Metodologia: foram utilizados 20 incisivos inferiores permanentes bovinos divididos em dois grupos $(\mathrm{n}=10)$. Em ambos os grupos foram feitas colagens de braquetes cerâmicos Allure utilizando-se Concise (3M Unitek) seguindo as recomendações do fabricante. Após a colagem, os espécimes foram mantidos em estufa, a $37^{\circ} \mathrm{C}$ por 24 horas, para completa polimerização do compósito. Após isso foi realizada a remoção dos braquetes, sendo que no grupo A foi utilizado alicate de corte de amarrilho e no grupo B alicate tipo How em braquete previamente fragilizado com broca diamantada. Em ambos os grupos o remanescente de compósito foi removido com broca de carboneto de tungstênio. Após descolagem, os espécimes foram preparados para análise em microscopia eletrônica de varredura, para posterior análise da superficie do esmalte. Resultados e Conclusões: os resultados mostraram maior quantidade de arranhões nos dentes do grupo $\mathrm{A}$, esses resultados foram estatisticamente superiores ao grupo $\mathrm{B}$ com $p<0,05$. Dessa maneira, verificou-se que o uso do alicate tipo How em braquete previamente fragilizado produziu menores arranhões à superfície de esmalte, indicando-o para uso clínico.
\end{abstract}

Palavras-chave: Remoção de braquetes ortodônticos. Topografia do esmalte. Alicates.

\section{INTRODUÇÃO}

A colagem direta de acessórios sobre a superfície dos dentes, sem a utilização de bandas, certamente agregou inúmeras vantagens aos tratamentos ortodônticos, entre elas: menor desconforto, simplicidade técnica, posicionamento mais preciso dos braquetes, melhor higienização, menor risco de cárie, diminuição de problemas periodontais e estética ${ }^{29}$.
No entanto, apesar de apresentarem estética melhorada, quando comparados aos aparelhos com bandas, os braquetes ortodônticos ainda continuaram antiestéticos, devido ao alto contraste entre o esmalte dentário e o metal do braquete.

A estética da aparatologia ortodôntica tem se tornado uma exigência crescente nos consultórios, especialmente entre adultos. Com objetivo de suprir tal demanda, surgiram os braquetes cerâmicos

* Especialista em Ortodontia pela Universidade Federal de Alfenas - UNIFAL. Mestre em Ortodontia pela Universidade Federal do Rio de Janeiro - UFRJ.

** Especialista em Ortodontia pela Universidade Federal de Alfenas - UNIFAL.

*** Mestre e Doutor em Ortodontia pela Universidade Federal do Rio de Janeiro - UFRJ. Professor adjunto de Ortodontia pela Universidade Federal do Rio de Janeiro - UFRJ. Professor do curso de especialização em Ortodontia da Universidade Federal de Alfenas - UNIFAL. 
que apresentam, como grande vantagem, a menor contraste com o esmalte dentário ${ }^{23}$.

Porém, sua utilização torna-se limitada, principalmente, devido ao seu alto custo, alta resistência do atrito à mecânica de deslizamento ${ }^{14,17,20}$ e danos iatrogênicos ao esmalte durante a sua remoção ${ }^{26}$, por produzir forte união na interface compósitobraquete ${ }^{21,22}$. Além disso, a impossibilidade de sofrerem distorções ${ }^{22}$ gera uma tensão demasiada na interface esmalte-compósito e, não raro, durante os procedimentos de descolagem pelas técnicas convencionais, ocorrem fraturas e danos importantes ao esmalte dentário ${ }^{7,13,15}$

Os objetivos da descolagem são remover o acessório e o compósito da superfície dentária usada na colagem e restaurar a superfície tão próximo quanto possível das condições de pré-tratamento, sem induzir danos iatrogênicos ${ }^{1,12,18}$. Para isto é necessária uma técnica correta.

Após a descolagem, é de extrema importância que tanto o acessório quanto a resina remanescente na superfície dentária sejam removidos sem produzir danos ao esmalte, de tal forma que essa superfície fique o mais semelhante possivel às condições apresentadas pré-tratamento ${ }^{4}$.

Vários métodos de remoção de braquetes são propostos, dentre esses citam-se: alicate removedor de braquetes número $347^{11}$, alicate tipo How $^{18}$, alicate removedor de bandas ${ }^{1}$, alicate de corte de amarrilho ${ }^{10}$, alicate indicado pelo fabricante $^{24}$, pistola removedora ${ }^{27}$, descolagem eletrotérmica ${ }^{25}$, utilização de ultra-som ${ }^{3}$ e 1 aser ${ }^{9}$.

A remoção do remanescente de compósito pode ser obtida por diferentes métodos, dentre eles: a utilização de alicate removedor de bandas ou um extrator ${ }^{10}$; uso de broca de carboneto de tungstênio $^{8,16}$; fresas de tungstênio carbide 30 lâminas ${ }^{4,10}$; broca de óxido de alumínio e finalização com broca multi-laminada, para complementação da remoção da resina mais próxima ao esmalte ${ }^{10}$; ou o uso de uma combinação de métodos ${ }^{5,11}$.

O objetivo dos autores do presente estudo foi avaliar a topografia do esmalte dentário após a descolagem de braquetes cerâmicos Allure (GAC/ Dentsply, New York, EUA) através de dois diferentes métodos.

\section{MATERIAL E MÉTODOS}

Foram utilizados 20 incisivos inferiores permanentes bovinos, armazenados em solução de formol a $10 \%$ e estocados em geladeira a $6^{\circ} \mathrm{C}$. A coroa destes dentes e pequena parte da raiz foram incluídas em resina acrílica auto-polimerizável, com o apoio de uma placa de vidro que serviu como planificador da face vestibular. Após a polimerização da resina, a face vestibular dos dentes foi lixada com lixas d'água, até que se conseguisse uma superfície de esmalte uniforme com diâmetro de aproximadamente $5 \mathrm{~mm}$.

Uma vez conseguida uniformidade da região do esmalte, foram realizados os procedimentos usuais de uma colagem, começando pela profilaxia com pedra-pomes e água por 10 segundos, utilizando-se micromotor de baixa rotação e taça de borracha. Em seguida, lavou-se pelo período de 10 segundos e, posteriormente, cada superfície foi seca com jatos de ar por 20 segundos. Uma vez realizada a profilaxia, lavagem e secagem, foi feito o condicionamento com ácido ortofosfórico (37\%) por 20 segundos, seguido de lavagem e secagem por igual tempo.

Após os procedimentos usuais de pré-colagem foi realizada a colagem propriamente dita, os espécimes foram colados com o compósito Concise 1929 (3M Unitek, São Paulo, Brasil), utilizando braquetes Allure (GAC/Dentsply, New York, EUA).

Os braquetes foram colados sendo mantido, pressionados por um determinado período, até que se completasse a polimerização. Durante o período que estavam sendo mantidos em posição, foram removidos os excessos de resina, com o auxílio de uma sonda exploradora (Duflex, Juiz de Fora, Brasil).

Após a colagem, os corpos-de-prova foram armazenados em água destilada e mantidos em 
estufa durante 24 horas, à temperatura de $37^{\circ} \mathrm{C}$. Ao final das 24 horas, os corpos-de-prova foram divididos aleatoriamente em dois grupos $(\mathrm{n}=10)$, sendo que no grupo A os braquetes foram removidos com alicate de corte de amarrilho (Fig. 1) e no grupo B com alicate tipo How (Fig. 2B) após ter sido realizada uma fragilização no longo eixo do braquete com broca diamantada (Fig. 2A), como proposto por Chevitarese e Ruellas ${ }^{6}$. Em ambos os grupos, após a remoção dos braquetes, o compósito foi removido com o uso de broca de carboneto de tungstênio ${ }^{8,16}$.

Após a descolagem, os espécimes foram preparados para análise da topografia dentária em microscopia eletrônica de varredura, com microscópio da marca JEOL, modelo SM-5800 LV (IME). Foi utilizado o aumento de 200x para a visualização da superfície de esmalte após remoção dos braquetes e do compósito remanescente.

Para avaliar a rugosidade propõe-se o Índice de Rugosidade do Esmalte (IRE), que avalia a superfície do esmalte quanto às suas condições de lisura. Para se aplicar este índice, a área fotografada foi subdividida em 100 partes iguais, com auxílio de uma grade criada em cima da fotografia com auxílio do programa de computador Power Point (Microsoft Corporation, Seattle, USA). Após isso, avaliou-se a quantidade de áreas danificadas durante o processo de remoção do braquete orto-

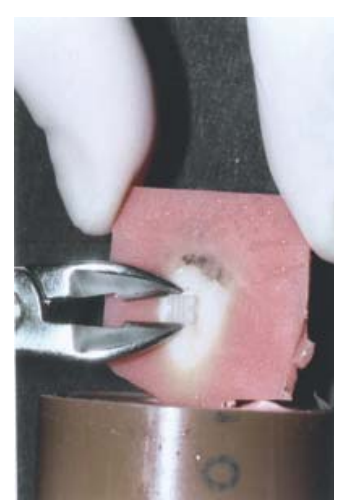

FIGURA 1 - Remoção do braquete com alicate de corte de amarrilho (grupo A) dôntico e do compósito residual. A avaliação foi realizada diretamente na tela do computador, em sala escura para melhor visualização da superfície do esmalte. Uma vez contadas as áreas danificadas, os espécimes foram classificados conforme o quadro 1 .

Desta forma, quanto maior a média do grupo maior serão os danos causados ao esmalte após a remoção.

A avaliação foi realizada em três períodos distintos, pelo mesmo indivíduo previamente calibrado. A média encontrada nas três avaliações foi anotada e classificada seguindo o IRE.

Esse indice possui maior fidedignidade quando comparado ao indice SRI (surface roughness index) proposto por Howell e Weekes ${ }^{12}$, uma vez que o IRE possui maior quantidade de escores.

\begin{tabular}{ll}
\hline 1 & até $10 \%$ da superfície arranhada \\
3 & até $20 \%$ da superfície arranhada \\
4 & até $30 \%$ da superfície arranhada \\
5 & até $40 \%$ da superfície arranhada \\
6 & até $50 \%$ da superfície arranhada \\
7 & até $60 \%$ da superfície arranhada \\
8 & até $70 \%$ da superfície arranhada \\
9 & até $80 \%$ da superfície arranhada \\
10 & até $90 \%$ da superfície arranhada \\
\hline
\end{tabular}

Quadro 1 - Índice de rugosidade do esmalte.
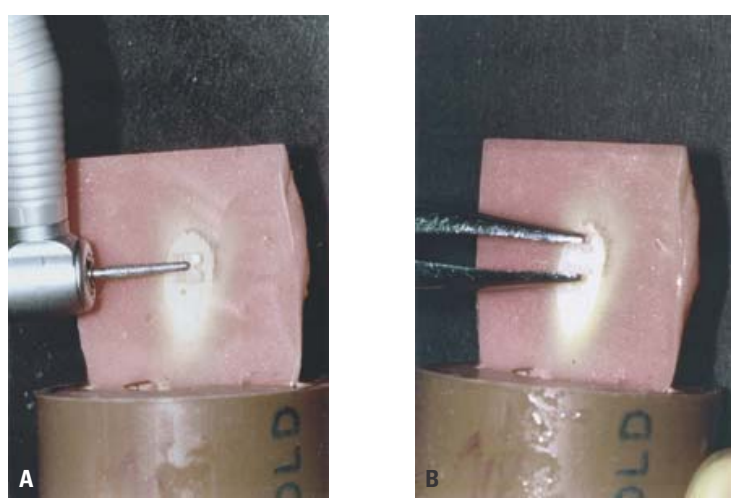

FIGURA 2 - A) Fragilização do longo eixo do braquete, previamente ao uso do alicate How. B) Posterior utilização do alicate tipo How. 
Uma vez analisada a superfície, os valores encontrados são levados à tabela para se determinar um escore adequado.

\section{RESULTADOS}

Os valores encontrados após classificação dos espécimes utilizando-se o Índice de Rugosidade do Esmalte (IRE) estão demonstrados na tabela 1.

$\mathrm{Na}$ avaliação do Índice de Rugosidade do Esmalte (IRE), houve diferença estatística $(\mathrm{p}<0,05)$ significante entre os grupos A e B ( $\mathrm{p}=0,00389051)$, como mostrado na tabela 2.

\section{DISCUSSÃO}

Desde a época de Edward Angle, os ortodontistas têm realizado pesquisas para encontrar formas para controlar mais precisamente os movimentos ortodônticos. Nos últimos anos, os ortodontistas têm dado mais atenção ao aspecto estético dos aparelhos ortodônticos dos quais se utilizam para realizar os movimentos dentários ${ }^{19}$. Não apenas o resultado final do tratamento ortodôntico é importante, o aspecto estético do paciente durante o mesmo tornou-se um fator de notável relevância ${ }^{1}$.

A busca por soluções estéticas aos aparelhos ortodônticos começou em 1965 com Newman, que introduziu acessórios plásticos tentando melhorar

Tabela 1 - Valores dos escores atribuídos a cada dente para o Índice de Rugosidade do Esmalte (IRE).

\begin{tabular}{rcc}
\hline IRE & A & B \\
\hline C1 & 9 & 6 \\
C2 & 9 & 3 \\
C3 & 9 & 6 \\
C4 & 8 & 3 \\
C5 & 8 & 2 \\
C6 & 5 & 5 \\
C7 & 6 & 6 \\
C8 & 4 & 2 \\
C9 & 5 & 4 \\
C10 & 5 & 5 \\
Média & 6,8 & 4,2 \\
\hline
\end{tabular}

a aparência dos aparelhos ortodônticos ${ }^{29}$. A aplicação clínica destes braquetes, no entanto, foi limitada, em razão de problemas encontrados quanto à durabilidade ${ }^{29}$, estabilidade dimensiona ${ }^{28}$, embebição por água e, como conseqüência, alteração de $\operatorname{cor}^{15}$. Tentando minimizar esses problemas apresentados, foram desenvolvidos, na década de 70 , braquetes confeccionados em cerâmica ${ }^{2,23}$.

No entanto, esses braquetes possuem limitações e dentre essas citam-se: alto custo, alta resistência do atrito à mecânica de deslizamento ${ }^{14,17,20}$ e danos iatrogênicos ao esmalte durante a sua remoção ${ }^{26,28}$, por produzir forte união na interface compósito-braquete ${ }^{21,22}$. Além disso, a impossibilidade de sofrerem distorções ${ }^{22}$ gera uma tensão demasiada na interface esmalte-compósito e, não raro, durante os procedimentos de descolagem pelas técnicas convencionais, ocorrem fraturas e danos importantes ao esmalte dentário ${ }^{7,13,15}$.

De acordo com os resultados obtidos pelo presente estudo, o grupo B, em que utilizou-se alicate tipo How após fragilização da braquete, apresentou superfície vestibular do esmalte com alguns arranhões (Fig. 4). No entanto, quando comparado ao grupo A, a condição do esmalte do grupo B foi mais compatível com a superfície de esmalte íntegro, corroborando com os achados de Zarrinia, Eid, Kehoe ${ }^{30}$; Campbel1 ${ }^{5}$; Gandini Jr. et al. ${ }^{10}$ e Braghetti ${ }^{4}$.

Deve-se ter, por parte do operador, habilidade manual e acuidade visual suficientes para não lesar o esmalte no momento da fragilizar o braquete, previamente ao uso do alicate de How. No momento em que a broca atingir o compósito, seu uso deve ser suspenso. Com a utilização do alicate How associado à broca diamantada, a possibilidade

Tabela 2 - Valores dos escores atribuídos a cada dente para o Índice de Rugosidade do Esmalte (IRE).

\begin{tabular}{ccc}
\hline grupos & $\begin{array}{c}\text { média } \\
\text { (desvio-padrão) }\end{array}$ & $\begin{array}{c}\text { análise estatística } \\
\text { (teste t) }\end{array}$ \\
\hline A & $6,8(1,988857852)$ & $\mathrm{a}$ \\
B & $4,2(1,619327707)$ & $\mathrm{b}$ \\
\hline
\end{tabular}




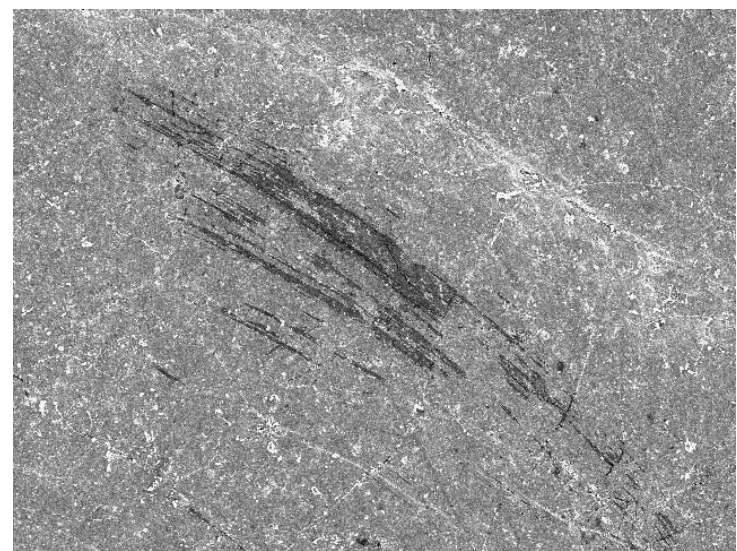

FIGURA 3 - Padrão de irregularidade da superfície do esmalte representativo do uso de alicate de corte de amarrilho, que recebeu escore 6 .

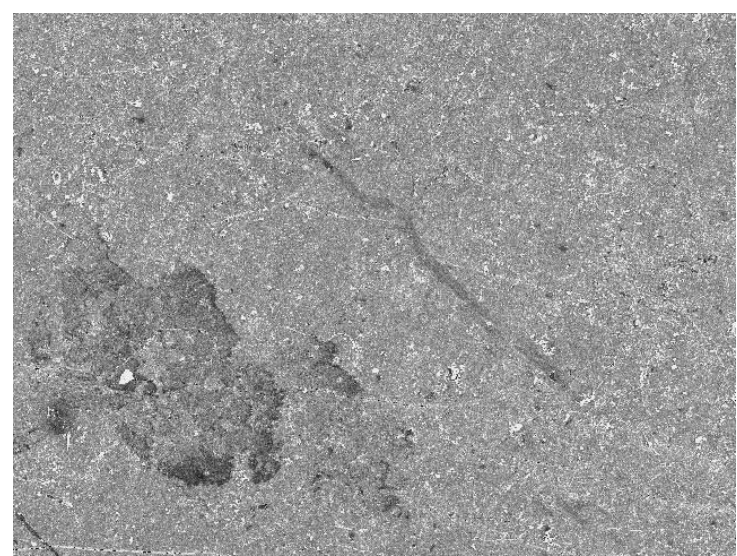

FIGURA 4 - Padrão de irregularidade da superfície do esmalte representativo do uso de alicate tipo How, que recebeu escore 4. de fratura do esmalte é praticamente nula.

O grupo A mostrou uma maior quantidade de arranhões, quando comparado (Fig. 3) ao grupo $\mathrm{B}$, resultado esse que corrobora com Braghetti ${ }^{4}$, quando do uso do alicate de corte.

Pelas figuras 3 e 4, pode-se verificar nitidamente a diferença no padrão de lisura da superfície do esmalte quando os dois métodos são usados. $\mathrm{Na}$ figura 3, está representada uma superfície do grupo A classificada como escore 6 e na figura 4 como escore 4.

\section{CONCLUSÃO}

A utilização do alicate de corte de amarrilho promove uma maior quantidade de arranhões ao esmalte, quando comparada ao uso do alicate tipo How com fragilização prévia do braquete.

Dessa maneira propõe-se o uso do alicate tipo How associado à fragilização prévia no longo eixo do braquete, para remoção de braquetes cerâmicos Allure (GAC/Dentsply, New York, EUA), e da broca de carboneto de tungstênio em baixa rotação para a remoção do remanescente de compósito.

\title{
Removal of ceramic brackets with How-type pliers in association with diamond drill - a topographic evaluation of the enamel
}

\begin{abstract}
Objective: to evaluate the topography of the tooth enamel after debonding Allure ceramic brackets using two different methods. Methods: Twenty bovine permanent lower incisors were divided into two groups $(n=10)$. In groups A and B Allure ceramic brackets were bonded to the teeth using Concise composite according to manufacture's recommendations. After the bonding procedures, the samples were kept in stove at $37^{\circ} \mathrm{C}$ for 24 hours for complete composite polymerization. Next, the brackets of group A were removed by using wire pliers, whereas the brackets of group $B$, which had been previously weakened with diamond drill, were debonded by using How-type pliers. In both groups the composite remnant was removed by using a tungsten carbide-tipped drill. Following the debonding procedures, the samples were prepared for scanning electronic microscopy so that their enamel surfaces could be analyzed. Results and conclusion: showing more scratch marks on the surfaces of the group A teeth were statistically superior to those found in group B $(p<0.05)$. Therefore, it was observed that the use of How-type pliers for debonding pre-weakened brackets caused less scratches on the enamel surface, which supports their clinical use.
\end{abstract}

Key words: Orthodontic bracket debonding. Enamel topography. Pliers. 


\section{REFERÊNCIAS}

1. BISHARA, S. E.; TRULOVE, T. S. Comparisons of different debonding techniques for ceramic brackets. An in vitro study. Part I. Background and methods. Am. J. Orthod. Dentofacial Orthop., St. Louis, v. 98, no. 3, p. 145-153, 1990.

2. BORDEAUX, J. M.; MOORE, R. N.; BAGBY, M. D. Comparative evaluation of ceramic bracket base designs. Am. J. Orthod. Dentofacial Orthop., St. Louis, v. 105, no. 6, p. 552-560, 1994

3. BOYER, D. B.; BISHARA, S. E. Debonding orthodontic ceramic brackets by ultrasonic instrumentation. Am. J. Orthod. Dentofacial Orthop., St. Louis, v. 108, no. 3, p. 262-266, 1995

4. BRAGHETTI, H. M. Estudo da eficiência de diferentes métodos de eliminação do remanescente de resina e polimento do esmalte dental, após a remoção de braquetes ortodônticos. 1999. Dissertação (Mestrado)-Faculdade de Odontologia de Araraquara, Universidade Estadual de São Paulo, Araraquara, 1999

5. CAMPBELL, P. M. Enamel surfaces after orthodontic bracket debonding. Angle Orthod., Appleton, v. 65, no. 2, p. 103-110, 1995

6. CHEVITARESE, O.; RUELLAS, A. C. O. Braquetes ortodônticos: como utilizá-los. 1. ed. São Paulo: Ed. Santos, 2005.

7. ELIADES, T.; VIAZIS, A. D.; LEKKA, M. Failure mode analysis of ceramic brackets bonded to enamel. Am. J. Orthod. Dentofacial Orthop., St. Louis, v. 104, no. 1, p. 21-26, 1993.

8. FRAUCHES, M. B.; CHEVITARESE, O. Descolagem de braquetes metálicos: efeito sobre a topografia do esmalte (in vitro). Rev. SOB, Rio de Janeiro, v. 2, n. 4, p. 110-118, 1993.

9. FRAUNHOFER, J. A.; VON ALLEN, D. J. Thermal effects associated with the Nd/YAG dental laser. Angle Orthod. Appleton, v. 63, no. 4, p. 299-303, 1993.

10. GANDINI JÚNIOR. L.G. et al. Avaliação de diferentes métodos de remoção de resina remanescente ao esmalte dentário após a descolagem de braquetes ortodônticos. Ortodontia, São Paulo, v. 28, p. 53-60, 1995

11. HONG Y. H.; LEW, K. K. K. Quantitative and qualitative assessment of enamel surface following five composite removal methods after bracket debonding. Eur. J. Orthod., London, v. 17 , no. 2 , p. 121-128, 1995

12. HOWELL, S.; WEEKES, W. T. An electron microscope evaluation of the enamel surface subsequent to various debonding procedures. Aust. Dent. J., Sydney, v. 35, no. 3, p. 245-252, 1990.

13. JEIROUDI, M. T. Enamel fracture caused by ceramic brackets. Am. J. Orthod. Dentofacial Orthop., St. Louis, v. 99, no. 2, p. $97-99,1991$

14. KEITH, O.; JONES, S. P.; DAVIES, E. H. The influence of bracket material, ligation force and wear on frictional resistance of orthodontic brackets. Br. J. Orthod., Oxford, v. 20, no. 2, p. 109-115, 1993

15. KRELL, K. V.; COUREY, J. M.; BISHARA, S. E. Orthodontic bracket removal using conventional ultrasonic debonding techniques, enamel loss, and time requirements. Am. J. Orthod. Dentofacial Orthop., St. Louis, v. 103, no. 3, p. 258-266, 1993.
16. OLIVER, R. G.; GRIFFITHS, J. Different techniques of residual composite removal following debonding - time taken and surface enamel appearance. Br. J. Orthod., London, v. 19 p. 131-137, 1992.

17. OMANA, H. M.; MOORE, R. N.; BAGBY, M. D. Frictional properties of metal and ceramic brackets. J. Clin. Orthod., Boulder v. 26, no. 7, p. 425-432, 1992.

18. RUELA, A. C. O.; CHEVITARESE, O.; GUIMARÃES, J. P.; ARAÚJO, M. T. S. Efeitos sobre a topografia do esmalte de dois métodos de descolagem de braquetes metálicos (in vivo). Rev. CROMG, Belo Horizonte, v. 3, n. 1, p. 1-5, 1997.

19. SCOTT JR., G. E. Fracture toughness and surface cracks: the key to understanding ceramic brackets. Angle Orthod., Appleton, v. 58 , no. 1 , p. $5-8,1988$

20. SPILLER, R. E.; DeFRANCO, D. J.; STORY, R. J. Friction forces in bracket-wire-ligature combinations. J. Dent. Res., New York, v. 69, p. $155,1990$.

21. STARLING, K. E. LOVE, B. J. Plasticization of adhesive to improve debonding of ceramic brackets. J. Clin. Orthod., Boulder, v. 4, no. 6, p. 319-322, 1993.

22. STROBL, K.; BAHUS, T. L.; WILLHAM, L.; BISHARA, S.E.; STWALLEY, W. C. Laser aided debonding of orthodontic ceramic brackets. Am. J. Orthod. Dentofacial Orthop., St. Louis, v. 101, no. 2, p. 152-158, 1992.

23. SWARTZ, M. L. Ceramic brackets. J. Clin. Orthod., Boulder, v. 22 , no. 2 , p. $82-88,1988$

24. THEODORAKOPOULOU, L. P.; SADOWSKY, P. L.; JACOBSON, A.; LACEFIELD JR., W. Evaluation of the debonding characteristics of 2 ceramic brackets: an in vitro study. Am. J. Orthod. Dentofacial Orthop., St. Louis, v. 125, no. 3, p. 329-336, 2004.

25. VARGAS, I. A. Descolagem eletrotérmica de brackets cerâmicos colados com diferentes compósitos. 1996. Dissertação (Mestrado)-Faculdade de Odontologia da Universidade Federal do Rio de Janeiro, Universidade Federal do Rio de Janeiro, Rio de Janeiro, 1996.

26. VIAZIS, A. D.; CAVANAUGH, G.; BEVIS, R. R. Bond strength of ceramic brackets under shear stress: an in vitro report. Am. J. Orthod. Dentofacial Orthop., St. Louis, v. 98, no. 3, p. 214-221, 1990.

27. VIEIRA, S. et al. Adesão em Ortodontia - Parte 3. J. Bras. Ortodon. Ortop. Facial, Curitiba, v. 7, n. 42, p. 466-472, 2002.

28. WINCHESTER, L. J. Bond strengths of five different ceramic brackets: an in vitro study. Eur. J. Orthod., London, v. 13, p. 293-305, 1991.

29. ZACHRISSON, B. U. Bonding in Orthodontic. In: GRABER, T. M.; WAIN, B. F. Orthodontic current principle and technique. 4rd ed. St. Louis: C. V. Mosby, 2005. p. 485-563.

30. ZARRINIA, K.; EID, N. M.; KEHOE, M. J. The effect of different debonding techniques on the enamel surface: an in vitro qualitative study. Am. J. Orthod. Dentofacial Orthop., St. Louis, v. 108, no. 3, p. 284-293, 1995.

\section{Endereço para correspondência}

Matheus Melo Pithon

Av. Otávio Santos 395 Centro Odontomédico $7^{\circ}$ andar, sala 705

CEP: 45.020-750 - Vitória da Conquista/BA

E-mail: matheuspithon@bol.com.br 